\title{
El rol del marketing urbano y la gobernanza de los gobiernos locales del Ecuador
}

\author{
The Role of Urban Marketing and Governance \\ of Local Governments in Ecuador \\ O papel do marketing urbano e a governança \\ dos governos locais do Equador
}

\author{
Stefany Cevallos \\ National University of Public Service. Budapest, Hungría \\ stefy220_@hotmail.com \\ https://orcid.org/0000-0003-1460-7324
}

DOI: https://doi.org/10.32719/25506641.2020.8.5

Recibido: 19 de febrero de 2020 - Revisado: 6 de marzo de 2020

Aceptado: 19 de abril de 2020

Artículo de investigación

Licencia Creative Commons

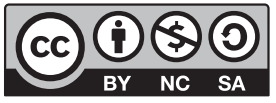




\section{Stefany Cevallos}

\section{Resumen}

Se analiza el rol del marketing urbano y la gobernanza de los gobiernos locales del Ecuador según el Código Orgánico de Organización Territorial, Autonomía y Descentralización (COOTAD). El debate de la gobernanza evidencia el potencial que pueden llegar a tener los gobiernos locales en asumir esa competencia por atraer Inversión Extranjera Directa (IED). La transversalización de la calidad integrada en este trabajo es producto del marketing urbano, en la construcción del branding o imagen del Ecuador y su posicionamiento económico en el ámbito regional e internacional. Se concluye que el gobierno local está coordinando agendas comerciales e institucionales en Alianzas Público-Privadas (APP) para asegurar el desarrollo económico, sociopolítico y tecnológico en el Ecuador.

Palabras clave: Gobernanza, marketing urbano, gobiernos locales, inversión extranjera directa, asociación público-privada.

JEL: H83 Administración pública; O38 Política pública.

\section{Summary}

The role of urban marketing and the governance of local governments in Ecuador is analysed according to the Organic Code of Territorial Organization, Autonomy and Decentralization (COOTAD). The governance debate shows the potential that local governments can have in assuming this competence to attract Direct Foreign Investment (FDI). The mainstreaming of integrated quality in this work is the product of urban marketing in the construction of the branding or image of Ecuador and its economic positioning in the regional and international arena. It is concluded that the local government is coordinating commercial and institutional agendas in Public-Private Alliances (PPP) to ensure economic, socio-political and technological development in Ecuador.

Keywords: Governance, urban marketing, local governments, foreign direct investment, publicprivate partnership.

JEL: H83 Public Administration; O38 Public policy.

\section{Resumo}

Procura-se analisar o papel do marketing urbano e a governança dos governos locais do Equador de acordo com o Código Orgânico de Organização Territorial, Autonomia e Descentralização (Código Órganico de Organización Territorial, Autonomía y Descentralización-COOTAD). O debate sobre a governança evidencia o potencial que podem chegar a ter os governos locais em assumir essa competição para atrair Investimento Estrangeiro Direto (IED). A transversalização da qualidade integrada em tal trabalho é produto do marketing urbano na construção do branding ou da imagem do Equador e de seu posicionamento econômico nos âmbitos regional e internacional. Conclui-se que o governo local está coordenando agendas comerciais e institucionais em Parcerias Público-Privadas (PPP) para assegurar o desenvolvimento econômico, sociopolítico e tecnológico no Equador. 
Palavras-chave: Governança, marketing urbano, governos/administrações locais, investimento estrangeiro direto, parceria público-privada.

JEL: H83 Administração pública; O38 Política governamental.

\section{Introducción}

L

a gobernanza está reemplazando constantemente modelos tradicionales de administración pública relacionados con jerarquías y mercados. Estudios de cognición social como Lee (1968) y Lynch (1960) señalan que las representaciones sociales tienden a estar conectadas con áreas específicas de la ciudad. En este sentido, se aborda el marketing urbano como un eje articulador de las políticas de los gobiernos locales y su desarrollo.

Para el presente trabajo se considera importante analizar cómo los gobiernos locales del Ecuador, bajo la modalidad de gestión asociación público-privada, conocida como APP, buscan ejecutar proyectos públicos con participación privada en calidad de contraprestación por su inversión y trabajo. Los gobiernos locales son considerados actores clave para la expansión internacional, pues incorporan la experiencia de un ente privado con el objeto de crear, desarrollar y operar una infraestructura pública y ofrecer un servicio.

Un factor determinante es la competitividad que puede llegar a tener el Ecuador frente a los mercados internacionales, como también la capacidad de mejorar significativamente su branding para poder anclarse a las necesidades de los países. Además, es importante considerar las ventajas de estar bajo el paraguas de la Organización de Naciones Unidas (ONU) y, a través de las Agencias de Promoción para Inversión en Latinoamérica y el Caribe (UNCTAD), hacer uso de la asesoría que los gobiernos locales disponen para cumplir lineamientos internacionales como los Objetivos de Desarrollo Sostenible (ODS) 2030. ${ }^{1}$ Además, entendiendo que una de las justificaciones centrales de los proyectos en relación a los gobiernos locales tiene que ver

1. La Agenda 2030 para el desarrollo sostenible contempla 169 metas y 17 objetivos. Entre estos, destaco pertinente al presente trabajo el 'Objetivo 8: trabajo decente y crecimiento económico'; 'Objetivo 11: ciudades y comunidades sostenibles' y 'Objetivo 17: alianzas para lograr los objetivos' (ONU 2015). El desarrollo como eje transversal y su carácter universal facilitará 
con reducir las brechas para el cumplimiento de los ODS, la UNCTAD pone de manifiesto que para tales efectos sería necesaria una combinación de inversión pública y privada local e internacional (IPA Observer UNCTAD 2019).

En este sentido, el objetivo del presente trabajo es visibilizar al marketing urbano y la gobernanza, aplicado a la internacionalización del Ecuador, como una propuesta para la calidad integrada en tanto estrategia de inclusión en el comercio mundial después del período del Socialismo del siglo XXI.

Para ello, el presente trabajo se compone de dos momentos. El primero se refiere al discurso teórico de la gobernanza, y cómo a través de los actores políticos se puede facilitar la dinámica de crear proyectos para el desarrollo del Ecuador en el aspecto social, económico, político, cultural y tecnológico. El segundo momento expone el rol del marketing urbano en la búsqueda por atraer inversión extranjera directa (IED), la que sería posible a través de la construcción de la imagen país.

Mediante los objetivos de análisis en la presente investigación, también se busca generar un aporte dentro de las líneas investigativas de la presente revista. Se espera contribuir al análisis de la gestión financiera, enfocándola en el ámbito de las finanzas públicas desde los gobiernos locales, y su relación con otro tipo de finanzas corporativas con respecto a las inversiones publico-privadas. Con ello, se podrán comprender las oportunidades de los entornos de los gobiernos locales para una mejor gestión financiera que permita el desarrollo económico, sociopolítico y tecnológico, tanto en el ámbito local como nacional.

\section{Metodología}

La investigación es explicativa y descriptiva, pues hace un repaso de las características locales y antecedentes sociales, políticos y económicos del Ecuador y su relación con la gobernanza y el marketing urbano. Sin embargo, es también exploratoria, puesto que las tendencias actuales y futuras de las políticas públicas ecuatorianas no pueden predecirse o confirmarse de inmediato.

la aplicación de dichos objetivos en la dinámica social, económica, política y cultural de las ciudades del Ecuador a través de las APP. 
Para esta investigación, se determinó la metodología por los métodos utilizados: base de datos primarios y secundarios, cuestionarios y análisis de datos.

\section{Gobernanza en el Ecuador}

El modelo de gobernanza hace referencia a las formas o modos de gobernar que involucran a actores no estatales (Levi-Faur 2012a). Cada modelo de gobernanza demanda un tipo específico de administración pública que se consolida mediante cambios enmarcados en una política de modernización de la administración pública. Este proceso requiere una gobernanza jerárquica que se someta a los actores involucrados y a la voluntad del gobierno, garantizando la autoridad del Estado (Kooiman 2002). El hecho de que los modelos de gobernanza no sean ciento por ciento puros en la realidad, ocasiona que los procesos de modernización de la administración pública tengan sus particularidades. En el caso ecuatoriano, los instrumentos desarrollados para modernizar la administración pública buscaban apalancar elementos de dos modelos de gobernanza distintos, el estatista y el liberal democrático; esto hace que el diseño de la política se enfrente a inconsistencias frente a los objetivos propios de cada modelo de gobernanza.

Levi-Faur (2012a), en el Oxford Handbook of Governance Highlights sobre el crecimiento de la gobernanza, señala que desde 1950 hasta 1979 ha habido un proceso lento de desarrollo y debate sobre este concepto. Sin embargo, cuando refiere a Oliver Williamson's Transaction Costs Economics: Governance of Contractual Relations (1979), destaca la evolución radical de la gobernanza, refiriéndose a este documento como uno de los más citados por la academia durante el período 1971-1980. Adicionalmente, Levi-Faur subraya la relevancia de los temas urbanos y educativos, que en la actualidad salen a la luz con más fuerza. Esto lo hace teniendo en cuenta que la educación y la gobernanza urbana son sectores directamente administrados por el gobierno, donde el Estado tiene un rol influyente en la dirección y el control de todo el proceso de desarrollo (2012).

Según Kooiman $(1993,3)$, la gobernanza "es un proceso de interacción político-social, que significa establecer una llamada tonalidad; creando las condiciones sociopolíticas para el desarrollo de los nuevos modelos de go- 
bierno interactivo en términos de cogestión, codirección y coorientación". Con respecto al papel del gobierno en dicho proceso, se sugiere que este sea altamente considerado, puesto que, por lo general, la capacidad del Estado es sobreestimada en la gobernanza.

De tal manera, es relevante señalar que los Estados tienen una autoridad que no posee ningún otro actor; elegir las reglas de juego del proceso, y cómo estructurar las condiciones que las moldean depende del mecanismo implementado para gobernar (Bell y Hindmoor 2009, 13). Además, "los patrones de gobernanza y los modos de gobernanza sociopolítica son el resultado de las interacciones públicas y privadas" (Kooiman 1993, 5).

Una mirada a la gobernanza tecnológica aconseja que las autoridades establezcan objetivos para sistemas de larga escala, y que los expertos técnicos y coordinadores implementen esas metas sociales en el diseño y la administración de aquellos (De Nardis 2014). Recapitulando la historia de la evolución de la industria, considerando los cuatro momentos de cambio paradigmático o de revoluciones industriales a lo largo de la historia hasta la actualidad, se establece que en la primera revolución, en la 1.0, se dio la generación de vapor y el primer telar mecánico; en la 2.0 la producción en cadena, la energía eléctrica y la primera cadena de montaje, mientras que la 3.0 se dio en el contexto de un mayor nivel de automatización de la electrónica y las tecnologías de información. Ahora bien, el evento social actual es característico del 4.0, en el cual existen sistemas ciber-físicos, industria y productos inteligentes, inteligencia artificial, Internet of Things Services, hiperconectividad y Big Data. En el ámbito de la gestión gubernamental, la digitalización es el principal motor del e-Governement, o gobierno electrónico, el cual deja traslucir una mirada bastante crítica sobre temas de gobernanza, pues es considerada como una herramienta de accountability o rendición de cuentas.

Así, la única constante de la gobernanza de internet es la condición de cambio constante, creando la incertidumbre omnipresente de un futuro alternativo rival. La autoridad en internet se involucra constantemente para abordar nuevos modelos de negocios, tecnologías emergentes y un contexto cultural cambiante (De Nardis 2014).

Antes de profundizar en los detalles de este trabajo y su nexo con el marketing urbano, es esencial adquirir una comprensión general del enlace entre 
gobierno, gobernanza y buen gobierno. El proceso es visto como un cambio del gobierno hacia la gobernanza, y actualmente hacia el buen gobierno. Como ponen de manifiesto González, Boza y León (2018), el concepto de gobernanza comienza a consolidarse en los años ochenta, vinculado al nuevo escenario y a las nuevas formas de entender el desarrollo de la época. Posteriormente, este evoluciona hasta que en el año 1994 el Banco Mundial define por primera vez al buen gobierno como:

Proceso de toma de decisiones previsible, abierto y fundamentado (es decir, un proceso transparente); una burocracia imbuida en valores; un brazo ejecutivo del gobierno fiable por sus acciones y una sociedad civil fuerte en la participación de los asuntos públicos; y todo ello bajo el imperio de la ley. (Banco Mundial 1994, citado en González, Boza y León 2018, 149)

En términos reales, en la mayoría de los países en desarrollo, como el Ecuador, el gobierno es quien direcciona estas dinámicas, por lo cual la gobernanza y el buen gobierno dependen en gran medida de este. En un sentido más amplio, el quién proporciona la gobernanza es la preocupación clave. Esto es así si consideramos el caso de los países en vías de desarrollo, especialmente en Latinoamérica, donde los Estados proporcionan el servicio público, siendo este último donde la gobernanza debería materializarse.

De esta forma, la gobernanza en las instancias locales connota un sentido de dirección en la capacidad y calidad integrada de acción del gobierno. Sin embargo, es difícil asignar una definición única a la gobernanza, ya que este término cambió de ser descriptivo a ser analítico. "La gobernanza es en sí misma el objeto de un debate teórico en el que se refleja la diversidad de tradiciones y corrientes en las ciencias sociales" (Fontaine 2010, 106).

Según la Constitución de la República del Ecuador (EC 2008), haciendo referencia al artículo 1 del Código Orgánico de Organización Territorial, Autonomía y Descentralización (COOTAD), se determina el modelo de administración, descentralización y desarrollo de los Gobiernos Autónomos Descentralizados (GAD) en el ámbito de la planificación, además de otras regulaciones del Estado en el ámbito nacional:

Art. 1.- Ámbito.- Este Código establece la organización político-administrativa del Estado ecuatoriano en el territorio: el régimen de los diferentes niveles de gobiernos autónomos descentralizados y los regímenes especiales, con el fin de garantizar su autonomía 
política, administrativa y financiera. Además, desarrolla un modelo de descentralización obligatoria y progresiva a través del sistema nacional de competencias, la institucionalidad responsable de su administración, las fuentes de financiamiento y la definición de políticas y mecanismos para compensar los desequilibrios en el desarrollo territorial. (COOTAD 2016, art. 1)

Los GAD son personas jurídicas de derecho público con autonomía política, administrativa y financiera. Los gobiernos locales tienen entonces la capacidad de formular y ejecutar decisiones públicas y, sobre todo, pueden generar vínculos estratégicos con otros actores públicos o privados.

En marzo de 2019 se eligieron 5.675 autoridades para los niveles de gobierno provincial (23 provincias), cantonal (221 cantones), parroquial (823 parroquias) y el régimen especial de Galápagos (CNE 2019). Estas autoridades de los gobiernos locales son quienes llevan a cabo las políticas públicas en territorio.

El gobierno central administra estrategias de gestión, pero el gobierno local, en su ámbito de acción, ajusta las decisiones políticas de manera más operativa y técnica para así poderlas confrontar con la ciudadanía. Este es un proceso de gobernanza donde ocurre el diálogo y la negociación con actores privados, además de los distintos grupos que tuvieran relación directa con los gobiernos locales por su interés de invertir.

\section{Marketing urbano}

Hay varias dimensiones recientes y contribuciones teóricas sobre el concepto de marketing urbano. Estas se desarrollaron desde finales de la década de 1980, cuando Ashworth (Ashwort y Voogd 1990), en Selling the City: Marketing Approaches in Public Sector Urban Planning, analizó por primera vez el fenómeno turístico urbano para comenzar a comprender el turismo en la ciudad y adoptar los estudios urbanos y sus críticas teóricas (Ashworth y Page 2011).

Son muchas las investigaciones progresivas que concluyen que el branding en ciudades respalda el significado de desarrollo y evolución (Green et al. 2016). Además, se considera que la aplicación del marketing urbano depende en gran medida de la construcción, comunicación y gestión de la 
imagen de la ciudad (Ashworth et al. 2015). Este es el caso de las ciudades intermedias (CEPAL 1998) de rango internacional, las cuales pueden posicionarse en el sistema de ciudades globales como centros de atracción basados en la calidad de vida urbana como su principal ventaja competitiva (Precedo Ledo et al. 2010).

La relación entre los gobiernos locales y el marketing urbano es entendida como la oportunidad y ventaja de tener una imagen de un país y de una ciudad específica, y los mecanismos mediante los cuales esa imagen puede redundar en una reputación favorable para el Ecuador. De acuerdo con Prats (2001, 103), "la gobernabilidad local debe ser entendida, esencialmente, como gobernabilidad urbana". Con ello, a su vez, tal como lo menciona Vásquez $(2014,161)$, se debe poder "identificar y reconocer las diferentes necesidades de la localidad y sus traducciones en una marca-ciudad, resultado de un acuerdo y un compromiso colectivo, que no solo debe buscar posicionar a la ciudad en el exterior a través de su imagen, sino también garantizar el fortalecimiento de la identidad local". En este sentido, la participación de los gobiernos locales se encuentra en el ámbito de la marca-ciudad, lo cual a su vez también puede llegar a tener impactos en un ámbito mayor en relación con la marca país.

Un ejemplo local con relación al marketing urbano en el sentido de la proyección de una imagen o marca específica de ciudad lo encontramos en Cuenca y su posicionamiento como un destino internacional de excelencia para el retiro de adultos mayores. Las investigaciones de Cabrera-Jara (2019a y 2019b) ponen de manifiesto la importancia del rol del marketing urbano por parte de las autoridades locales cuencanas para el posicionamiento de Cuenca como ciudad prometida para jubilados norteamericanos mediante la explotación de sus atractivos turísticos y un sostenido desarrollo inmobiliario.

Para los gobiernos locales de países en vías de desarrollo, una positiva asociación con gobiernos de otros países, empresas y organizaciones internacionales marca un hito de proyección y reputación. En este sentido, al asegurar su posicionamiento económico, el Ecuador, al igual que otros países de América del Sur, buscan aumentar la influencia de la IED en su desarrollo. Esta búsqueda se da, no solo atrayéndola en la forma de grandes cantidades 
de dinero, sino también beneficiándose de ella en áreas como tecnología, empleo, exportaciones, cualificación y en general competitividad.

Si bien la economía del Ecuador aún sigue dependiendo ampliamente de la producción del petróleo, dada la volatilidad en sus precios en los últimos años, desde 2015 dejó de depender exclusivamente de la producción de aquella (BCE 2015). ${ }^{2}$ Por ejemplo, de acuerdo con la Organización Mundial del Comercio $(2019,19)$, "La participación de las exportaciones de petróleo en las exportaciones totales disminuyó del $57 \%$ en 2013 al 36\% en 2017". De manera similar, datos del Banco Central del Ecuador acerca del valor añadido bruto (VAB) de las actividades petroleras, frente a las no petroleras, muestran una marcada disminución de las segundas a partir de fines del 2014, tendencia que se acentúa en el segundo trimestre de 2015, cuando tienen una disminución de $-4,3 \%$, mientras que el $\mathrm{VAB}$ petrolero mantiene un saldo positivo (BCE 2015).

A la par, en actividades económicas no tradicionales (a la exportación petrolera, del banano y el camarón) como el turismo, se ha podido ver una transformación de una actividad incipiente, a una de mayor importancia, lo que podría ilustrar la importancia del marketing urbano y la gobernanza de los gobiernos locales. Estudios como el de Barzola y Contreras (2018) demuestran en el caso ecuatoriano una relación favorable entre los ingresos por turismo internacional al país y el crecimiento del PIB, y con ello ser una fuente directa de aporte al crecimiento económico del Ecuador.

Sobre la base de esta riqueza y de sus ventajas comparativas, Ecuador logró desarrollar varias industrias de exportación en la década de los noventa, basándose sobre todo en productos básicos con posibilidades de IED. Adicionalmente, el marco legal del Ecuador establece políticas estables que ofrecen confianza y beneficios a los inversores extranjeros.

2. En el contexto de las medidas de confinamiento y aislamiento ante el covid-19, la economía ecuatoriana fue afectada por una caída significativa en los precios de su principal producto de exportación, el petróleo crudo, y un menor dinamismo en la demanda externa de productos no petroleros, resultado de la contracción económica de China, Estados Unidos y la Unión Europea. En el mes de marzo de 2020, el petróleo ecuatoriano se cotizó en promedio en USD 23,0 por barril. 


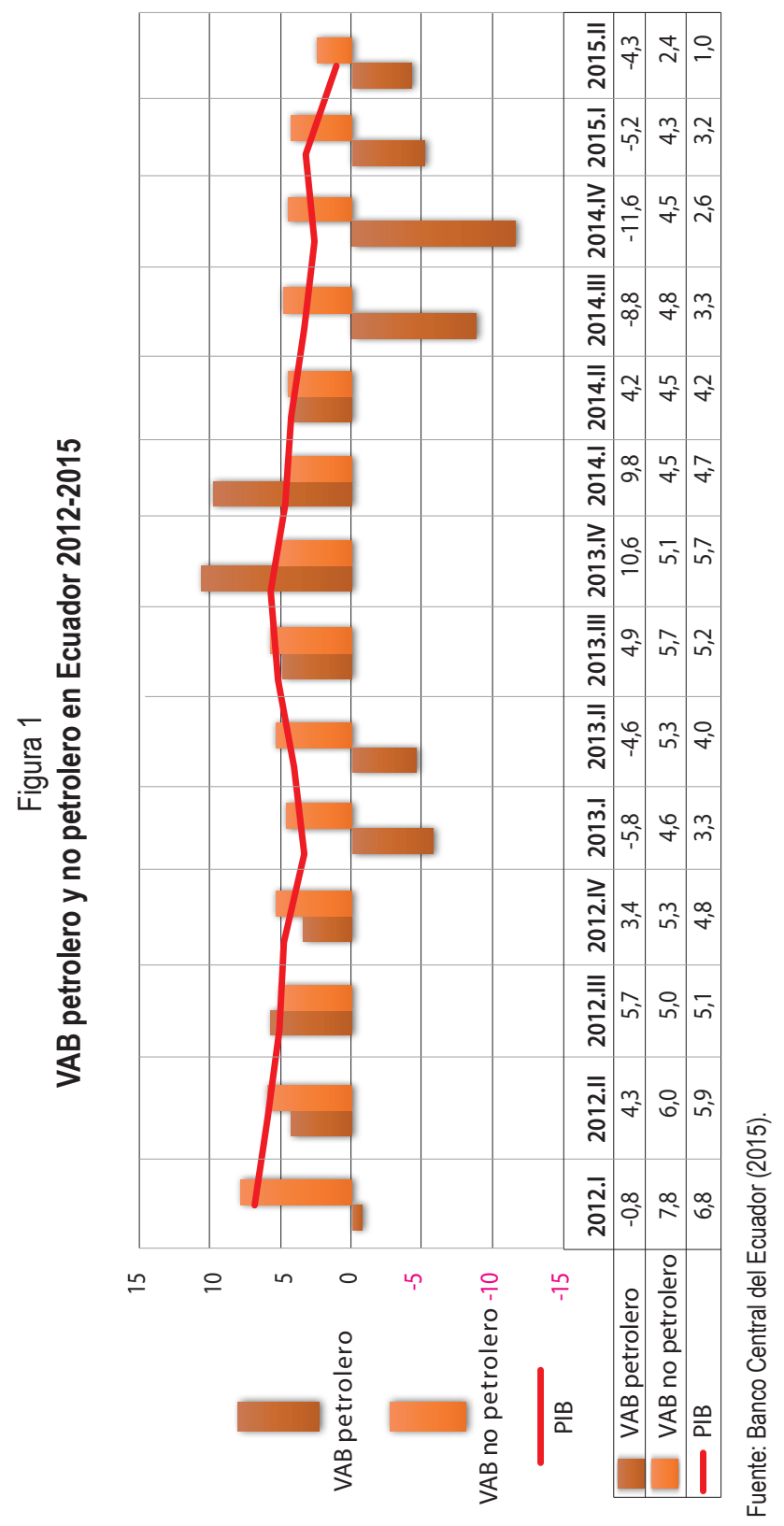


Ecuador cuenta con la Ley Orgánica de Incentivos para Asociaciones Público-Privadas y la Inversión Extranjera (EC 2015), que tiene por objeto establecer incentivos para la ejecución de proyectos, $y$, en términos generales, promover el financiamiento productivo, la inversión nacional y la inversión extranjera en el Ecuador. Además, Ecuador cuenta también con una ley específica: Ley Orgánica para el Fomento Productivo, Atracción de Inversiones, Generación de Empleo, y Estabilidad y Equilibrio Fiscal (EC 2018). Para Keeble (2019), dicha ley se presenta como un gran incentivo de atracción para las inversiones extranjeras en el país.

La Constitución de la República del Ecuador (EC 2008) y el marco legal correspondiente establecen que la gestión de la cooperación internacional es competencia de los gobiernos subnacionales. En este contexto, estos últimos ya han tenido experiencia trabajando con agencias de cooperación internacional, a través de las cuales se han realizado contribuciones a los procesos de desarrollo local. Como ejemplo tenemos el trabajo efectuado con la Conferencia de las Naciones Unidas sobre Comercio y Desarrollo (CNUCYD) y sus agencias de promoción de inversiones.

Las agencias de promoción de inversiones son las instituciones responsables de promover la inversión extranjera en un área específica. Este tipo de instituciones pueden ser organizaciones gubernamentales sin fines de lucro, o incluso entidades privadas dirigidas por juntas directivas que pueden incluir funcionarios gubernamentales y gerentes comerciales. Por lo tanto, una de las principales actividades de una agencia de promoción de inversiones es el posicionamiento del país en el mercado internacional, una tarea que implica la construcción de una imagen favorable, y con esto, la creación de una marca país.

Es importante señalar que la ONU ofrece apoyo a la promoción de inversión para el desarrollo sustentable de las ciudades, y que los gobiernos locales son entes imprescindibles para asegurar los ODS 2030. En el ámbito de la participación APP, son pertinentes los siguientes objetivos: ODS 8: empleo digno y crecimiento económico; ODS 11: ciudades y comunidades sostenibles; y ODS 17: alianzas para lograr los objetivos.

Este contexto económico nos permitirá concluir que el país tiene muchas atracciones para los inversionistas extranjeros, las cuales, con políticas apropiadas, pueden transformarse en oportunidades. Además, Ecuador tiene abun- 
dantes recursos naturales no renovables tales como los minerales inexplorados. Sus recursos renovables como el banano y el plátano verde, las flores naturales, los peces, los camarones, el abacá, el cacao, entre otros, bosquejan un perfil económico atractivo para el mercado regional e internacional.

\section{Balance preliminar}

Todo país quiere vender su identidad única y asegurar su lugar en el mapa. El actor estrella en este proceso es, sin lugar a duda, el gobierno. A través de su buen gobierno, así como también de sus esfuerzos de persuasión global, podrá construir una imagen que, consecuentemente, se refleje en opiniones, ideas y, finalmente, decisiones de inversión.

La marca país ofrece oportunidades de negocio para propiciar una apertura internacional con un saldo positivo para el Ecuador, y debería ser asumida por el gobierno central y los gobiernos locales. La esfera política tiene total conocimiento de que el gobierno en todos sus niveles es el único ente que puede vender dicha marca.

El Doing Business Rankings 2019 ubica al Ecuador, de acuerdo a la facilidad que ofrece para hacer negocios, en el rango 123 a nivel mundial y 21 a nivel de Latinoamérica y el Caribe, de entre 42 países. En el tercer lugar, después de México y Puerto Rico, está Colombia, y de acuerdo a ProColombia, la Agencia de Promoción de Inversiones Invest in Bogotá coordinó y ejecutó el proyecto Movistar Arena Bogotá o estadio El Campín. Este proyecto fue construido por la empresa TuBoleta y el grupo chileno HLR, bajo el paraguas de una APP, con el asesoramiento de Investment Promotion Agencies (IPA) de la ONU. El mencionado proyecto es considerado como un ejemplo de IED y consecución de los ODS 8, 11 y 17 (IPA Observer UNCTAD 2019).

Lo que pasa en el país vecino no está lejos de la política de Estado del período del Gobierno ecuatoriano (2017-2021), que constituyó la promoción de inversiones a través del Comité Estratégico de Promoción y Atracción de Inversiones. Actualmente, Pro Ecuador es la institución que tiene la tarea de promocionar al país a través de oficinas consulares para que productos como el banano y el plátano, el camarón, las flores naturales, entre otros, se consoliden en el mercado internacional. 
Figura 2

Clima de inversiones y negocios en países de América Latina

(Ponderaciones neutrales)

\begin{tabular}{|c|c|c|c|}
\hline \multicolumn{3}{|l|}{ Posición } & Puntaje sobre 100 \\
\hline & 1 & Perú & 87 \\
\hline & 2 & Bahamas & 85 \\
\hline & 2 & Barbados & 85 \\
\hline & 4 & República Dominicana & 84 \\
\hline & 5 & Jamaica & 80 \\
\hline & 6 & Ecuador & 78 \\
\hline & 7 & Panamá & 75 \\
\hline & 7 & Trinidad y Tobago & 75 \\
\hline & 9 & Brasil & 74 \\
\hline & 10 & El Salvador & 72 \\
\hline & 11 & Chile & 71 \\
\hline & 12 & Guatemala & 69 \\
\hline & 13 & Uruguay & 68 \\
\hline & 14 & Argentina & 57 \\
\hline & 14 & Costa Rica & 57 \\
\hline & 16 & Nicaragua & 54 \\
\hline & 17 & Colombia & 52 \\
\hline & 18 & Honduras & 47 \\
\hline & 19 & México & 45 \\
\hline & 20 & Paraguay & 37 \\
\hline & 21 & Venezuela & 3 \\
\hline & & Promedio & 65 \\
\hline MADU & $\mathrm{O}(80$ & & \\
\hline DESAF & OLLA & $(60-79)$ & \\
\hline EMERC & ENTE & $-59)$ & \\
\hline NAC & : (C & & \\
\hline
\end{tabular}

Puntaje del 0 al 100 en donde 100 es mejor. Ranking entre los 21 países de $A L C, 1=$ mejor, = antes de una posición significa un empate

Fuente: Infrascopio (2019). 
Según el ranking Infrascopio, herramienta informativa e índice de referencia que evalúa la capacidad de los países de América Latina y el Caribe para llevar a cabo APP, Ecuador fue considerado un país naciente en estas alianzas (Infrascopio 2014). Sin embargo, las mejoras en el clima de inversiones y negocios impulsaron puntuaciones más altas en esta categoría para Ecuador, ubicándolo como un país desarrollado en APP (Infrascopio 2019). Ecuador ocupa el sexto lugar en la región, habiéndose beneficiado de un cambio en el respaldo político a favor de las APP desde 2015, así como de incentivos fiscales para promoverlas, que se aprobaron con amplio respaldo político a un país a escala comparativa con otros países vecinos.

Dos ejemplos de lo arriba mencionado. El primero es el Puerto de Posorja en la provincia de Guayas. Este proyecto, que inició en 2016 y que fue ejecutado conjuntamente por el sector público y privado bajo la figura de APP, fue la primera experiencia de este tipo de alianzas desde la promulgación de la Ley Orgánica de Incentivos para Asociaciones Público-Privadas y la Inversión Extranjera (EC 2015). Esta asociación permite oportunidades de crecimiento para la comunidad de Posorja, ya que entró en funcionamiento en 2019, y es considerado de gran impacto en la economía local y nacional en tanto los acuerdos firmados permiten que la inversión sea totalmente privada, excluyendo riesgos financieros para el Estado ecuatoriano. A su vez, al ser un puerto de aguas profundas, y emblemático para la región y el mundo, su construcción influiría positivamente en la imagen y proyección del país en el ámbito internacional.

El segundo ejemplo es el GAD Provincial de Santo Domingo de los Tsáchilas, que bajo la modalidad de APP, persigue su proyecto de construir un puerto seco en la ciudad de Santo Domingo (GADPSDT 2019).

Una breve lectura socioeconómica del Ecuador, en relación a la gobernanza y al marketing urbano, permite señalar, en primera instancia, que el gobierno nacional y los gobiernos locales, ya sean provinciales o municipales, se enfrentan a múltiples e independientes cambios a la hora de gestionar sus recursos. Estos cambios apuntan al objetivo de crear oportunidades para los ciudadanos, reducir la pobreza, mejorar las condiciones de vida de la población y, por ende, mantenerse en el poder o buscar una reelección.

Sin embargo, persiste el acalorado debate sobre los efectos de la IED en el desarrollo (Te Velde 2003). Los defensores de la IED argumentan que esta 
es buena para el desarrollo, y, por lo tanto, perciben su rápida expansión en América Latina como un maná del cielo (Vodusek 2002). En algunos casos, los críticos sostienen que la IED conduce a un aumento de la pobreza, el aislamiento y el abandono de las capacidades locales (Vial 2001).

\section{Conclusiones}

Este trabajo concluye con la perspectiva de la acción política del Ecuador, y en específico de la gobernanza local, con el fin de atraer inversión extranjera directa. Para ello, se presentó el rol del marketing urbano y la gobernanza con el objetivo de operativizar su definición y las prácticas gubernamentales de los gobiernos locales.

El análisis de los distintos componentes y características del marketing urbano y la gobernanza en Ecuador podrían coadyuvar en la búsqueda de espacios para el consenso respecto a temas de inversión extranjera, como también a la toma de decisiones en la política del país. Por esta razón, se espera que el presente documento sea un pequeño aporte al ambiente de diálogo necesario para la gobernanza y el marketing urbano del Ecuador.

Existen dos procesos, imagen y branding, que experimentan mayor crecimiento en el marketing urbano para el desarrollo de la ciudad. En consecuencia, en el caso de los proyectos Puerto de Posorja y Puerto Seco de Santo Domingo de los Tsáchilas, se está produciendo una valoración en los activos inmateriales, al ponerse en marcha nuevos procesos de participación política y social por parte de los gobiernos locales.

Finalmente, el marketing urbano, descrito en conjunto, puede ser una opción para la construcción de una imagen del Ecuador que asegure el desarrollo en todo su concepto, desde el fortalecimiento de la imagen ciudad fomentada por los gobiernos locales. Esta imagen deber ser la que transversalice la calidad integrada en el país de la mano de la gobernanza y el buen gobierno. 


\section{Referencias}

Ashworth, Gregory, y Henk Voogd. 1990. Selling the City: Marketing Approaches in Public Sector Urban Planning. Londres: Belhaven Press.

Ashworth, Gregory, y Mihalis Kavaratzis. 2007. "Beyond the Logo: Brand Management for Cities. Journal of Brand Management 16 (8): 520-531. 10.1057/palgrave.bm.2550133.

Ashworth, Gregory, y Page Stephen J. 2011. "Urban Tourism research: Recent Progress and Current Paradoxes". Tourism Management 32 (1): 1-15. 10.1016/j.tourman.2010.02.002.

Banco Interamericano de Desarrollo. 2020. “APP en la región servicios de asesoría 2020”. Accedido 12 de enero. https://bit.ly/2xJHZAY.

Bell, Stephen, y Andrew Hindmoor. 2009. Rethinking Governance, the Centrality of the State in Modern Society. Nueva York: Cambridge University Press.

Cabrera-Jara, Natasha. 2019a. "Gentrification in Latin American Heritage Areas: Ethical Questioning Based on the Case of Cuenca, Ecuador". Urbe. Revista Brasileira de Gestão Urbana (11): 1-15. https://doi.org/10.1590/2175-3369.011.e20180201.

---. 2019b. "Real Estate Market and Urban Metamorphosis in Intermediary Cities. Gringolandia in Cuenca: The Promised Land". Bitácora Urbano Territorial 29 (1): 91-100. https:// doi.org/10.15446/bitacora.v29n1.75223.

Carnap, Rudolf. 1950. Logical Foundations of Probability. Chicago: University of Chicago Press.

Castells, Manuel. 1968. "Y a-t-il une sociologie urbaine?". Sociologie du travail (10): 72-90. https://bit.ly/3h7hBD7.

CO Pro Colombia. 2017. "Invest in Bogotá 2017”. Accedido 4 de enero de 2020. https://bit. ly/3eAgudG.

Comisión Económica para América Latina y el Caribe (CEPAL) y Ministero degli Affari Esteri Cooperazione Italiana (MAE). 1998. Ciudades intermedias de América Latina y el Caribe: propuestas para la gestión urbana, compilado por Ricardo Jordán y Daniela Simioni. Santiago: EPAL. https://bit.ly/30XLMGV.

Conferencia de las Naciones Unidas sobre Comercio y Desarrollo (UNCTAD). 2014. "Informe Nacional sobre las Exportaciones Verdes (INEVs)". Accedido 4 de enero de 2020. https://bit.ly/2VN4WLu.

DeNardis, Laura. 2014. The Global War for Internet Governance. Connecticut: Yale University.

Doing Business. 2020. "Rankings Business in Latin America and Caribbean". Accedido 25 de enero. https://bit.ly/2XPWj5z.

EC. 2008. Constitución de la República del Ecuador. Registro Oficial 449, 20 de octubre.

---.2010. Código Orgánico de Organización Territorial, Autonomía y Descentralización (COOTAD). Registro Oficial, Suplemento 303, 19 de octubre de 2010.

---. 2015. Ley Orgánica de Incentivos para Asociaciones Público-Privadas y la Inversión Extranjera (2015). Registro Oficial, Suplemento 652, 18 de diciembre. 


\section{Stefany Cevallos}

---. 2018. Ley Orgánica para el Fomento Productivo, Atracción de Inversiones, Generación de Empleo, y Estabilidad y Equilibrio Fiscal. Registro Oficial 309, 21 de agosto.

EC Banco Central de Ecuador (BCE). 2016. IT-2019 IVT Versión 6. "Manual de Balanza de Pagos y Posición de Inversión Internacional". Accedido 12 de febrero de 2020. https://bit. ly/2VlTmba.

EC Consejo Nacional Electoral. 2017. "Transparencia 2017”. CNE. Accedido 12 de enero de 2020. https://bit.ly/2Vot7Rr.

EC DP World. 2018. "Desarrollo Local Posorja 2018”. Accedido 12 de febrero de 2020. https://bit.ly/2VIYzcb.

EC Gobierno Autónomo Descentralizado Provincial de Santo Domingo de los Tsáchilas (GADPSDT). 2020. "APP Puerto Seco Santo Domingo". Accedido 7 de enero. https://bit.ly/ 34R4EaA.

EC Instituto Nacional de Estadística y Censos. 2010. "Evolución de las variables investigadas en los Censos de Población y Vivienda del Ecuador 1950, 1962, 1974, 1982, 1990, 2001 y 2010". Accedido 12 de febrero de 2020. https://bit.ly/2BhYwgV.

EC Pro Ecuador. 2020. "Exportaciones en Ecuador IED 2020”. Accedido 12 de enero. https:// bit.ly/34UFFU2.

Fontaine, Guillaume. 2010. Petropolítica: una teoría de la gobernanza energética. Quito: FLASCO.

Gert-Jan Hospers. 2010. "Lynch's the Image of the City after 50 Years: City Marketing Lessons from an Urban Planning Classic". European Planning Studies 18 (12): 2073-2081. 10.1080/09654313.2010.525369.

González, Matías, José Boza y Javier de León. 2018. "Buen gobierno y eficacia de la ayuda al desarrollo". Criterio Libre 16 (29): 143-162. 10.18041/1900-0642/criteriolibre.2018v16n 29.5012 .

Green, Amalia, Debra Grace y Helen Perkins. 2016. "City Branding Research and Practice: An Integrative Review". The Journal of Brand Management 23 (3): 252-272. https://doi. org/10.1057/bm.2016.8.

Institute for Identity. 2019. "Social Image in Cities" Visual Style: Business and Investment. Accedido 12 de enero de 2020. https://bit.ly/2RSPbSl. Accedido 12 de enero de 2020. https://bit.ly/2Kk03Ev.

Keeble, James Eduard. 2019. "Promoting Investment and Developing Production in Ecuador". Revista Internacional Jurídica y Empresarial (2): 151-156. https://bit.ly/2LJ7UMs.

Kooiman, Jan. 1993. Modern Governance, New Government-Society Interactions. Londres: Sage Publications.

---. 2002. "Governance. A Social-Political Perspective". En Participatory Governance. VS Verlag für Sozialwissenschaften, editado por Jürgen R. Grote y Bernard Gbikpi, 71-96. https://doi.org/10.1007/978-3-663-11003-3_4.

Levi-Faur, David. 2012a. The Oxford Handbook of Governance. Nueva York: Oxford University Press. 
---. 2012b. From “Big Government” to "Big Governance”? The Oxford Handbook of Governance. Berlín: Aufbau Verlag. doi: 10.1093/oxfordhb/9780199560530.013.0001.

Lynch, Kevin. 1960. The Image of the City. Cambridge: The MIT Press.

Organización de Naciones Unidas (ONU). 2015. Resolución A/RES/70/1 Transformar nuestro mundo: la Agenda 2030 para el Desarrollo Sostenible. Accedido 1 de enero de 2020. https://bit.ly/3boZyF7.

Organización Mundial del Comercio (OMC). 2019. Examen de las políticas comerciales WT/ $T P R / G / 383$ - Ecuador. Accedido 28 de enero de 2020. https://bit.ly/3dikaiu.

Popper Karl. 1959. The Logic of Scientific Discovery. Londres: Hutchinson.

Prats, Joan. 2001. "Gobernabilidad democrática para el desarrollo humano. Marco conceptual y analítico". Instituciones y desarrollo 10: 103-148. https://bit.ly/37UHeT8.

Precedo Ledo, Andrés, José Orosa González y Alberto Míguez Iglesias. 2010. "De la planificación estratégica al marketing urbano: hacia la ciudad inmaterial”. EURE 36 (108): 5-27. http://dx.doi.org/10.4067/S0250-71612010000200001.

Rosero Barzola, Christian Xavier, y Cristina Zúñiga Contreras. 2018. "Análisis de la hipótesis del crecimiento del turismo: caso Ecuador”. Comercio y Negocio (5): 77-85. https://bit. ly/2AlJ6bf.

Te Velde, Dirk Willem. 2003. Foreign Direct Investment and Income Inequality in Latin America: Experiences and Policy Implications Overseas Development Institute. Londres: Legend Press.

The Economist Intelligence Unit. 2019. INFRASCOPIO 2019 Evaluando el entorno para las asociaciones público-privadas en América Latina y el Caribe, comisionado por el Banco Interamericano de Desarrollo. Nueva York. Accedido 18 de enero de 2020. https://bit. ly/2BybYxg.

UNCTAD. 2019. Promoting Investment for Sustainable Development in Cities. Accedido 18 de enero de 2020. https://bit.ly/2RUL2x3.

Vásquez Ornés, Sandra. 2014. “La gestión urbana sostenible: conceptos, rol del gobierno local y vinculación con el marketing urbano". Provincia (31): 147-171. https://bit.ly/2LQD5p3.

Vial, Joaquín. 2001. "Foreign Investment in the Andean countries". CID discussion paper. Cambridge: Harvard University Center for International Development. https://bit.ly/2Z0DprM.

Vodusek, Ziga. 2002. Foreign Direct Investment in Latin America: The role of European Investors. Madrid: Inter-American Development Bank Rumagraf.

World Bank. 2018. "APP in Latin America and Caribbean”. Accedido 18 de enero de 2020. https://bit.ly/2zgEi6g. 